\title{
Sputum induction for the diagnosis of tuberculosis
}

\author{
A M A Shata, J B S Coulter, C M Parry, G Ching'ani, R L Broadhead, C A Hart
}

\begin{abstract}
Confirmation of tuberculosis in young children is difficult as they seldom expectorate sputum. Gastric aspirates are invasive and stressful and like laryngeal swabs are seldom smear positive. Induction of sputum by nebulised hypertonic saline (3\%) was attempted in 30 Malawian children aged 3-15 years and was successful in 29. Four sputa were smear positive and Mycobacterium tuberculosis was cultured from three of them. A further four sputa were culture positive though smear negative. In all, the diagnosis of tuberculosis was confirmed in eight (28\%) of 29 children. The presence of polymorphonuclear cells in the specimen was indicative of sputum, in contrast to epithelial cells which originate from saliva. A predominance of polymorphonuclear cells in specimens was more common in older children and these specimens were more likely to be smear positive or culture positive. Sputum induction is a useful method for the confirmation of tuberculosis and is possible in young children.

(Arch Dis Child 1996;74:535-537)
\end{abstract}

Keywords: tuberculosis, sputum induction, diagnosis.

The diagnosis of tuberculosis in children is based on clinical grounds combined with a chest $x$ ray and a tuberculin test, and sometimes through identification and/or culture of Mycobacterium tuberculosis when required. However, the tuberculin test may be negative in several conditions including malnutrition, disseminated tuberculosis, and acute viral infections such as measles. The diagnosis of tuberculosis is now complicated by the advent of HIV infection which is associated with an increased prevalence of tuberculosis, a reduced sensitivity to the tuberculin test, and difficulty with interpreting chest $x$ rays, particularly in children. ${ }^{1}$ Malawi has, as have many other sub-Saharan countries in Africa, experienced an upsurge in tuberculosis notification since 1985 (Malawi National Tuberculosis Control Programme, 1993). Children with tuberculosis, or other pulmonary infection, usually do not expectorate sputum but swallow it. It is thus generally presumed that it is not possible to obtain sputum from particularly young children.

The technique of sputum induction using hypertonic saline was originally described in the diagnosis of pulmonary tuberculosis in adults. ${ }^{2}$ It is commonly used for the diagnosis of Pneumocystis carinii pneumonia in adults with AIDS $^{3-5}$ and not until recently in children. ${ }^{6}{ }^{7}$ The technique was found to be useful for the diagnosis of tuberculosis in adult Malawians. ${ }^{8}$ We have therefore studied the use of sputum induction in Malawian children with suspected pulmonary tuberculosis.

\section{Methods}

Patients admitted to the children's ward or seen in the outpatient department at Queen Elizabeth Central Hospital, Blantyre, Malawi, who were suspected of having tuberculosis, were studied. The children were under 15 years of age and verbal consent from their parents was obtained. The diagnosis of pulmonary tuberculosis was based on history, clinical examination, chest $x$ ray, and a Mantoux test (10 units). The presence and size of the BCG scar was documented in each case. Induction was undertaken once and preferably on the fasting child, or at least it was not performed after meals or snacks. The children were encouraged to clean their mouths and remove debris. Sputum was induced by nebulising $5-10 \mathrm{ml}$ of $3 \%$ sterile saline for $10-20 \mathrm{~min}$ using an ultrasonic nebuliser (Mistogen EN145). ${ }^{4}$ After use the Mistogen nebuliser equipment was thoroughly washed and then soaked in glutaraldehyde overnight. Care was taken in handling specimens because of their infectious nature.

\section{LABORATORY METHODS}

A smear was made and examined by ZiehlNeelsen (ZN) stain and acid auramine phenol for acid fast bacilli. The smears were also examined under low power $(\times 100)$ and a semiquantitative assessment made as to whether epithelial or polymorphonuclear cells predominated on the film. The sputum was processed by the modified Petroff technique and cultured on Lowenstein Jensen media at $37^{\circ} \mathrm{C}$ for eight weeks in Malawi. An aliquot of the sample was frozen at $-20^{\circ} \mathrm{C}$ for transportation to the department of medical microbiology, University of Liverpool. In Liverpool, the samples were recultured for mycobacteria. All mycobacterial isolates were identified by standard methods. ${ }^{9}$

\section{Results}

Fifty seven children with tuberculosis were selected and sputum induction was undertaken in 30 . It was successful in $29(97 \%)$. The only 
Table 1 Relation between age and sputum quality

\begin{tabular}{lcccc}
\hline Cells & $3-4$ & $5-9$ & \multicolumn{2}{c}{$10-15$} \\
& Years & Years & Years & Total \\
\hline $\begin{array}{l}\text { Mainly epithelial } \\
\begin{array}{l}\text { Mixed polymorphs/ } \\
\text { epithelial }\end{array}\end{array}$ & 3 & 0 & 1 & 4 \\
$\begin{array}{l}\text { Mainly polymorphs } \\
\text { Total }\end{array}$ & 3 & 1 & 7 & 11 \\
\hline
\end{tabular}

child in whom it failed was 4 years of age and did not have a cough. Reasons for not attempting sputum induction in the remainder included refusal (7), too young to cooperate (18; all of these were under 3 years), and too ill (2). None of the children had a productive cough

Of the 29 children, 11 (38\%) were 3-4 years, seven $(24 \%)$ were $5-9$ years, and $11(38 \%)$ were 10-15 years of age. Three to five millilitres of hypertonic saline $(3 \%)$ were found to be sufficient to induce sputum and 10-20 minutes nebulisation was adequate. The induction was tolerated well by the children (and parents). There were no side effects or complications. A few children complained of an unpleasant taste or nausea but no vomiting was recorded.

Epithelial cells were detected more often in younger children, suggesting the samples contained saliva; conversely older children were more likely to have polymorphs, indicative of good quality sputum (table 1).

Four out of 29 sputum samples (14\%) were smear positive on $\mathrm{ZN}$ stain. In three of these four specimens, acid-alcohol-fast bacilli (AFB) were scanty and in one they were detected in large numbers, resembling adult smears. Three of these four positive sputa were culturepositive. A further four sputa were smear negative but culture positive. Thus the total numbers which were either smear positive or culture positive, or both, were eight out of $29(28 \%)$. Nocardia asteroides, which are aerobic Gram positive and weakly acid fast bacilli, were isolated in one child. There was a direct relation between sputum quality (that is, the number of polymorphs) and culture positivity. Six of the 11 sputa with a high proportion of polymorphs were smear positive or culture positive, or both (table 2). The seven sputa which were culture positive in Malawi were

Table 2 Relation between sputum quality and results of AFB smear and culture

\begin{tabular}{|c|c|c|c|c|c|}
\hline Cells & $\begin{array}{l}\text { Smear- } \\
\text { Culture - }\end{array}$ & $\begin{array}{l}\text { Smear + } \\
\text { Culture - }\end{array}$ & $\begin{array}{l}\text { Smear- } \\
\text { Culture + }\end{array}$ & $\begin{array}{l}\text { Smear }+ \\
\text { Culture }+\end{array}$ & Total \\
\hline Mainly epithelial & 4 & 0 & 0 & 0 & 4 \\
\hline Polymorphs/epithelial & 12 & 1 & 1 & 0 & 14 \\
\hline Mainly polymorphs & 5 & 0 & 3 & 3 & 11 \\
\hline Total & 21 & 1 & 4 & 3 & 29 \\
\hline
\end{tabular}

Table 3 Diagnosis of tuberculosis (TB) in 29 children

\begin{tabular}{llll}
\hline & Confirmed $T B^{\star}(n=8)$ & Unconfirmed TB $(n=21)$ & Total $(n=29)$ \\
\hline Family history & 8 & 18 & 26 \\
BCG scar & 7 & 20 & 27 \\
Mantoux 5-10 mm & 2 & 7 & 9 \\
Mantoux $>10 \mathrm{~mm}$ & 1 & 2 & 3 \\
Chest $x$ ray=likely & 8 & 19 & 27 \\
TB & & & \\
\hline
\end{tabular}

* AFB on smear and/or culture.

† Lymphadenopathy, TB or widespread consolidation, collapse or cavities. confirmed in Liverpool. They had been frozen for up to eight weeks.

Twenty seven children (93\%) had a BCG scar (table 3). Only one of the eight children with confirmed tuberculosis had a Mantoux response of $>10 \mathrm{~mm}$ and two had a response of 5-10 mm. Overall only three children had a Mantoux response of $>10 \mathrm{~mm}$ (table 3 ).

\section{Discussion}

Sputum induction was undertaken in the 30 children suspected of tuberculosis and able to cooperate, and was successful in 29. Sputa were either $\mathrm{ZN}$ smear positive or culture positive, or both, in eight $(28 \%)$. The procedure was possible in children as young as 3 years and was found to be safe, practical, and well tolerated. Eleven $(38 \%)$ of the children were 3-4 years of age (table 1 ).

In adults, it is recommended that sputum induction should be avoided in ill patients, especially those with AIDS, due to possibility of arterial desaturation ${ }^{10}$ and a rapid increase in volume of pre-existing pleural effusion. ${ }^{11}$ No adverse effects were noted in our patients, even in two children with pleural effusions. For ethical reasons we were unable to determine the HIV status of our patients.

Positive $\mathrm{ZN}$ smears are more likely to be obtained from induced than expectorated sputum. ${ }^{8}$ Droplets of the nebulised saline are deposited in the lung peripheries, which, because of the hypertonicity, draw interstitial fluid into the lower airway by osmosis. ${ }^{5}$ Fluid produced by the nebulised saline mobilises material in the lower airways, and the repeated coughing - stimulated by hypertonic salinemoves the material into the upper airways whence it is then expectorated. Evidence that the origin of material expectorated during sputum induction is from the lower airways is shown by the fact that $P$ carinii (usually present in the alveoli) is rarely detected in expectorated sputum, while it is often found in induced sputum. Because of the infectivity of induced sputum, care should be taken in handling the material, which may contain a variety of pathogenic organisms including $M$ tuberculosis. ${ }^{12} \mathrm{~N}$ asteroides, which is a recognised complication of HIV infection, was isolated from one patient whose chest $x$ ray mimicked that of tuberculosis.

The other alternatives to induced sputum for the diagnosis of tuberculosis are gastric aspirate and a laryngeal swab. These, and especially laryngeal swabs, are seldom positive on direct smear ${ }^{13}{ }^{14}$ and thus culture is required. In children with pulmonary tuberculosis, $M$ tuberculosis has been cultured from gastric aspirate in $33 \%,{ }^{15} 36 \%,{ }^{16}$ and $39 \%,{ }^{17}$ and a higher proportion in infants $(75 \%),{ }^{17}{ }^{18}$ whereas only $2 \%$ or less may be positive on gastric aspirate smears. ${ }^{15}$ Lloyd reported positive culture using laryngeal swabs in $63 \%$ and gastric aspirate in $28 \%$ of 60 children with various forms of pulmonary and non-pulmonary tuberculosis. ${ }^{14}$ If non-pulmonary tuberculous cases were excluded, the proportion of culture positive children would probably have been higher. In 20 children with pulmonary tuberculosis sub- 
jected to both bronchoalveolar lavage and gastric lavage, $M$ tuberculosis was cultured from only two of the former and $10(50 \%)$ of the latter. ${ }^{19}$ All were smear negative. A comparison of induced sputum with gastric aspirate in adults found that if gastric aspiration was undertaken after induction of sputum, specimens were equally culture positive but gastric aspiration was inferior if undertaken before induction of sputum. ${ }^{2}$ Gastric aspiration is distressing to the child, and mothers (and nurses) of some ethnic backgrounds in Africa are very resistant to its use on their child. The advantage of induced sputum is the higher probability of obtaining a positive smear. However, culture is the optimal method for confirmation of tuberculosis and it will identify the species and sensitivity of the mycobacterium. There are no comparisons of culture between sputum induction and gastric aspiration in children.

In industrialised countries the diagnosis of tuberculosis is usually based on a tuberculin test and a chest $x$ ray; also BCG is regarded as providing protection against pulmonary tuberculosis. ${ }^{20}$ Table 3 shows the contrast with Malawi: the Mantoux test response was more than $10 \mathrm{~mm}$ in only one child and was between 5 and $10 \mathrm{~mm}$ in only two children with confirmed tuberculosis. We do not know their HIV status. Seven (87\%) of eight children with confirmed, and 20 (95\%) of 21 with suspected, tuberculosis had a BCG scar (table 3).

Sputum induction is a safe, useful investigation in children suspected of tuberculosis and can be successfully employed in children as young as 3 years of age. Sputum induction offers the possibility of obtaining a positive smear and in older children and adolescents could be used as a screening test. If the specimen is smear negative, and particularly if it has a high proportion of epithelial cells (indicating saliva), induction should be repeated. A direct comparison of sputum induction with gastric aspiration and laryngeal swabs is required to estimate optimal methods for smear and culture in children. Presently, gastric aspirate remains the method of choice for culture in young children, particularly infants.
We thank the senior medical superintendent and staff of the paediatric wards and laboratories of the Queen Elizabeth Central Hospital, Blantyre, for their helpful cooperation. Mycobacterial aspirates were confirmed by the Mycobacterial Reference Unit, Public Health Laboratory, University Hospital, Cardiff.

The work was submitted by AMAS in partial fulfilment of requirements for the Master in Tropical Paediatrics examination at Liverpool School of Tropical Medicine.

1 Harries $\mathrm{AD}$. Tuberculosis and human immunodeficiency virus infection in developing countries. Lancet 1990; $\mathrm{i}$ : 387-90.

2 Carr DT, Karlson AG, Stilwell GG. A comparison of induced sputum and gastric washing in the diagnosis of tuberculosis. Mayo Clin Proc 1957; 42: 23-5.

3 Bigdy TD, Margolskee D, Curtis JL, et al. The usefulness of induced sputum in the diagnosis of Pneumocystis carinii in patients with acquired immunodeficiency syndrome. Am Rev Respir Dis 1986; 133: 515-8.

4 Leigh TR, Parsons P, Hume C, Hussain OAN, Gazzard BG, Collins JV. Sputum induction for the diagnosis of Pneumocystis carinii pneumonia. Lancet 1989; ii: 205-6.

5 Miller RF, Leigh TR, Collins JV, Mitchell DM. Tests giving an etiological diagnosis in pulmonary disease in patients infected with HIV. Thorax 1990; 45: 62-5.

6 Ognibene FP, Gill VJ, Pizzo PA, et al. Induced sputum to diagnose Pneumocystis carinii pneumonia in immunosup-

7 Foot ABM, Caul EO, Roome AP, Oakhill A, Catterall JR. An assessment of sputum induction as an aid to diagnosis of respiratory infections in the immunocompromised child. $f$ Infect 1992; 24: 49-54.

8 Parry CM, Kamoto O, Harries AD, et al. The use of sputum induction for establishing a diagnosis in patients with suspected pulmonary tuberculosis in Malawi. Tuber Lung Dis 1995; 76: 72-6.

9 Collins $\mathrm{CH}$, Grange JM, Yates MD. Organisation and practice in tuberculosis bacteriology. London: Butterworth, 1985.

10 Miller RF, Buckland J. Semple SJG. Arterial desaturation in HIV positive patients undergoing sputum induction. Tho$\operatorname{rax} 1991$; 46: 449-51.

11 Nelson M, Bower M, Smith D, et al. Life threatening complications of sputum induction. Lancet 1990; 335: 112-3.

12 Centers for Disease Control. Mycobacterium tuberculosis transmission in a health clinic-Florida 1988. MMWR 1989; 38: 256-64.

13 Crofton J, Horne N, Miller F. Clinical tuberculosis. London: Macmillan Education, 1992: 193.

14 Lloyd AVC. Bacteriological diagnosis of tuberculosis in children. A comparative study of gastric lavage and laryngeal swab methods. East Afr Med 1968; 45: 140-43.

15 Barnett GD, Styblo K. Bacteriological and x-ray status of tuberculosis following primary infection acquired during adolescence or later. Bull IUAT 1977; 52: 5-15.

16 Morrison JB. Natural history of segmental lesions in primary pulmonary tuberculosis. Arch Dis Child 1973; 48: $90-8$.

17 Starke JR, Taylor-Watts KT. Tuberculosis in the pediatric population of Houston, Texas. Pediatrics 1989; 84: 28-35.

18 Vallejo JG, Ong LT, Starke JR. Clinical features, diagnosis and treatment of tuberculosis in infants. Pediatrics 1994; 84: 1-7.

19 Abadco DL, Steiner P. Gastric lavage is better than bronchoalveolar lavage for isolation of Mycobacterium tuberculosis in childhood pulmonary tuberculosis. Pediatr tuberculosis in childhood pulm

20 Colditz GA, Brewer TF, Berkey CS, et al. Efficacy of BCG vaccine in the prevention of tuberculosis. $\mathscr{f} A M A$ 1994; 271: 698-702. 